\title{
VOLUME 52, 2008
}

\section{CONTRIBUTOR INDEX}

\section{A}

Abascal-Agorreta M (see Vera-Alvarez et al). 2008;52: 264-266 (Letter)

Abdali K (see Shamsi et al). 2008;52:187-190

Abdul-Karim FW (see Farag et al). 2008;52:294-296

Abnet CC (see Pan et al). 2008;52:14-23

Abraham EK (see Ramadas et al). 2008;52:396-398 (Letter)

Adán A (see Saro et al). 2008;52:87-90

Afarid M (see Mostaghni et al). 2008;52:597-601

Aisner S (see Fitzhugh et al). 2008;52:240-246

Akbulut M, Zekioglu O, Kapkac M, Erhan Y, Ozdemir N. Fine Needle Aspiration Cytology of Glycogen-Rich Clear Cell Carcinoma of the Breast: Review of 37 Cases with Histologic Correlation. 2008;52:65-71

Akbulut M, Zekioglu O, Ozdemir N, Kapkac M. Fine Needle Aspiration Cytology of Mammary Carcinoma with Choriocarcinomatous Features: A Report of 2 Cases. 2008;52:99-104

Aktepe F (see Tokyol et al). 2008;52:235-239

Al-Abbadi M (see Vella et al). 2008;52:377-378 (Letter)

Al-Abbadi MA (see Feng et al). 2008;52:434-438

Al-Agha OM, Khader SN, Cajigas A, Blank W, Grafstein N, Seymour AW. Fine Needle Aspiration of Urethral Recurrence of Urothelial Carcinoma After Radical Cystectomy Presenting as a Perineal Mass: A Case Report. 2008;52:94-98

Al-Ayadhy B (see Kapila et al). 2008;52:681-686

Al-Mosawy FA (see Kapila et al). 2008;52:681-686

Al-Sebeih K (see Palmieri et al). 2008;52:691-696

Aledavud A (see Daneshbod et al). 2008;52:387-389 (Letter)

Ales A (see Terčelj et al). 2008;52:584-590

Almeida JD, Lima CF, Brandão AAH, Cabral LAG. Evaluation of Staining Methods for Cytologic Diagnosis of Oral Lesions. 2008;52:697-701

Altay M (see Demir et al). 2008;52:309-312

Alvarez-Santín C. Endometrial Adenocarcinoma: Prevention and Early Diagnosis. 2008;52:748 (Book Review)

Amadori PL (see Bonzanini et al). 2008;52:541-548

Amano S (see Komatsu et al). 2008;52:591-596

Ambaye AB (see Sherman et al). 2008;52:659-664

Anand M, Sharma S, Kumar R, Raina V. Diagnostic Considerations in Prolymphocytes in Pleural Fluid: A Case Report. 2008;52:251-254
Ang L-C (see Keith et al). 2008;52:260-263 (Letter) Angeloni C (see Maccallini et al). 2008;52:568-574 Anshu (see Gabhane et al). 2008;52:354-356 Anshu (see Gangane et al). 2008;52:325-328 Anshu (see Gangane et al). 2008;52:619-622 Antonelli C (see Maccallini et al). 2008;52:568-574 Apice G (see Fulciniti et al). 2008;52:612-618 Arabi MA (see Daneshbod et al). 2008;52:268-270 (Letter)

Argüelles M (see González et al). 2008;52:490-494

Armbruster C, Bernhardt K, Setinek U. Pulmonary

Tumorlet: A Case Report of a Diagnostic Pitfall in Cytology. 2008;52:223-227

Aron M (see Mathur et al). 2008;52:740-743 (Letter) Arora VK (see Mathur et al). 2008;52:740-743 (Letter) Arundhati (see Garbyal et al). 2008;52:204-206 Arundhati (see Ghosh et al). 2008;52:733-735 Ashfaq R (see Patino et al). 2008;52:718-720 Ashraf MJ (see Azarpira et al). 2008;52:220-222 Ashraf MJ, Azarpira N, Vasei M, Tavakol MH, Khademi B. Thyroid Paraganglioma: Diagnostic Pitfall in Fine Needle Aspiration Biopsy. 2008;52: 745-747 (Letter)

Assiri AH (see Mokhtar et al). 2008;52:169-177

Asthana AK (see Ghosh et al). 2008;52:733-735

Athanassiadou P, Grapsa D. Value of Endoscopic Retrograde Cholangiopancreatography-Guided Brushings in Preoperative Assessment of Pancreaticobiliary Strictures: What's New? 2008;52:24-34

Azarpira N (see Ashraf et al). 2008;52:745-747 (Letter) Azarpira N, Ashraf MJ, Shishegar M. Fine Needle Aspiration Findings in Angiofollicular Hyperplasia with Eosinophilia: A Case Report. 2008;52:220-222

\section{B}

Babu KS, Goel D, Prayaga A, Rao IS, Kumar A. Intraabdominal Hydatid Cyst: A Case Report. 2008; 52:464-466

Babu S (see Goel et al). 2008;52:344-346

Badhe BA (see Siddaraju et al). 2008;52:378-380 (Letter)

Badhe BA (see Siddaraju et al). 2008;52:495-499

Baek HJ (see Jin et al). 2008;52:357-360

Bafa M (see Pappa et al). 2008;52:485-489

Bal A (see Kumar Behera et al). 2008;52:500-504

Bandyopadhyay S (see Feng et al). 2008;52:434-438

Banerjee SK (see Patino et al). 2008;52:718-720 
Bangerter M, Brudler O, Heinrich B, Schrembs I, Griesshammer M. Open or Closed Nuclear Membrane? A Question to Help Distinguish Malignant Lymphoma from Carcinoma and Sarcoma. 2008; 52:52-55

Bargaje A, Venkataraman G, Wojcik EM. Associated Cytologic Findings on Pap Tests from Patients with Genital Herpes: A Retrospective Cohort Analysis in 82 Cases Over 11 Years. 2008;52:512514 (Letter)

Barnes PJ (see MacIntosh et al). 2008;52:530-534

Barroca H, Correia C, Castedo S. Cytologic and Cytogenetic Diagnosis of Pediatric Renal Cell Carcinoma Associated with $\mathrm{t}(\mathrm{X} ; 17)$. 2008;52:384-386 (Letter)

Barroca H, Marques C, Candeias J. Fine Needle Aspiration Cytology Diagnosis, Flow Cytometry Immunophenotyping and Histology in Clinically Suspected Lymphoproliferative Disorder: A Comparative Study. 2008;52:124-132

Basu A, Sistla SC, Siddaraju N, Verma SK, Iyengar KR, Jagdish S. Needle Tract Sinus Following Aspiration Biopsy of Papillary Thyroid Carcinoma: A Case Report. 2008;52:211-214

Batra M, Handa U, Mohan H, Sachdev A. Comparison of Cytohistologic Techniques in Diagnosis of Gastroesophageal Malignancy. 2008;52:77-82

Batra VV, Jain S, Singh DK, Kumar N. Cytomorphologic Spectrum of Giant Cell Tumor of Tendon Sheath. 2008;52:152-158

Bedayat GR (see Daneshbod et al). 2008;52:72-76

Behera B (see Kumar Behera et al). 2008;52:500-504

Behera SK, Nayak S, Mishra D. Diagnosis by Fine Needle Aspiration of Disseminated Cutaneous Rhinosporidiosis Presenting as Cutaneous and Subcutaneous Tumor-like Nodules. 2008;52:635636 (Letter)

Bellas C (see Yuste et al). 2008;52:191-195

Bentz J (see Layfield et al). 2008;52:273-275 (Letter)

Bergman S (see Patel et al). 2008;52:454-463

Bernhardt K (see Armbruster et al). 2008;52:223-227

Bhatia P, Dey P, Uppal R, Shifa R, Srinivasan R, Nijhawan R. Cell Blocks from Scraping of Cytology Smear: Comparison with Conventional Cell Block. 2008;52:329-333

Bhatia P, Srinivasan R, Rajwanshi A, Nijhawan R, Khandelwal N, Wig J, Vasishtha RK. 5-Year Review and Reappraisal of Ultrasound-Guided Percutaneous Transabdominal Fine Needle Aspiration of Pancreatic Lesions. 2008;52:523-529

Bhattacharjee MB (see Takei et al). 2008;52:445-450

Bhujun KH (see Garbyal et al). 2008;52:204-206

Bibbo M (see Jacob-Ampuero et al). 2008;52:687-690

Bibbo M (see Young et al). 2008;52:277-278

Bishop JW (see Pacheco et al). 2008;52:575-578
Bisht SP (see Kishore et al). 2008;52:710-712 Blank W (see Al-Agha et al). 2008;52:94-98

Bobhate SK (see Wahane et al). 2008;52:381-384 (Letter)

Bohra A (see Garbyal et al). 2008;52:204-206

Bohra A (see Garbyal et al). 2008;52:270-273 (Letter)

Bonzanini M, Amadori PL, Sagramoso C, Dalla Palma P. Expression of Cytokeratin 19 and Protein p63 in Fine Needle Aspiration Biopsy of Papillary Thyroid Carcinoma. 2008;52:541-548

Boon ME (see Fulciniti et al). 2008;52:178-186

Botti G (see Fulciniti et al). 2008;52:178-186

Botti G (see Fulciniti et al). 2008;52:612-618

Boudreaux C (see Carter et al). 2008;52:725-728

Boukovinas I (see Sioutopoulou et al). 2008;52:304-308

Bove P (see Fulciniti et al). 2008;52:612-618

Brainard J (see Weber et al). 2008;52:320-324

Brandão AAH (see Almeida et al). 2008;52:697-701

Brimo F, Nahal A. Malignant Epithelioid Hemangioendothelioma with Spindle Phenotype: Report of an Unusual Case Diagnosed by Fine Needle Aspiration Cytology. 2008;52:721-724

Brudler O (see Bangerter et al). 2008;52:52-55

Budhwar P (see Goel and Budhwar). 2008;52:424-433

Budhwar P (see Goel and Budhwar). 2008;52:602-606

Bukvic M (see Kemp et al). 2008;52:471-474

Bulten J (see Grefte et al). 2008;52:35-44

Bundele MM (see Siddaraju et al). 2008;52:109-113

Burstein DE (see Xiao and Burstein). 2008;52:118119 (Letter)

Cabral LAG (see Almeida et al). 2008;52:697-701

Cajigas A (see Al-Agha et al). 2008;52:94-98

Cancellieri A (see Trisolini et al). 2008;52:263-264 (Letter)

Candeias J (see Barroca et al). 2008;52:124-132

Cappellari JO (see Li et al). 2008;52:607-611

Caraceni D (see Maccallini et al). 2008;52:568-574

Carreras A (see Saro et al). 2008;52:87-90

Carter JE, Evans TN, Tucker JA. Cytologic Diagnosis of Alveolar Soft Part Sarcoma of the Lower Extremity by Fine Needle Aspiration and Correlation with Core Biopsy: A Case Report. 2008;52:459463

Carter JE, Nelson JJ, Eves M, Boudreaux C. Diagnosis of Linitis Plastica-Type Gastric Adenocarcinoma by Endoscopic Ultrasound-Guided Fine Needle Aspiration: A Case Report. 2008;52:725-728

Casadio C (see Miller et al). 2008;52:207-210

Castedo S (see Barroca et al). 2008;52:384-386 (Letter)

Catalina-Fernández I, Sáenz-Santamaria J, LópezPresa D. Fine Needle Aspiration Cytology of Ganglioneuroma. 2008;52:380-381 (Letter)

Caudill JL, Humphrey SK, Salomão DR. Islet Cell 
Tumor of the Pancreas: Increasing Diagnosis After Instituting Ultrasonography-Guided Fine Needle Aspiration. 2008;52:45-51

Cavuoti D (see Patino et al). 2008;52:718-720

Cavuoti D (see Rust et al). 2008;52:475-480

Cazzaniga M (see Miller et al). 2008;52:207-210

Chachra KL (see Halder et al). 2008;52:286-293

Challa S (see Prayaga et al). 2008;52:702-709

Chandler N (see Steinman et al). 2008;52:279-285

Chang H, Sun C-F. Detection of B Cell Lymphoma Involvement in $\mathrm{T}$ Cell-Rich Serous Fluid by Immunoglobulin Gene Rearrangement: A Report of 2 Cases. 2008;52:231-234

Chang T-C (see Hsieh et al). 2008;52:361-365

Charoenkwan K, Nimmanahaeminda K, Khunamornpong S, Srisomboon J, Thorner PS. Effects of Gel Lubricant on Cervical Cytology. 2008;52: 654-658

Chattwal PK (see Tewari et al). 2008;52:743-745 (Letter)

Chen KTK. Cytology of Cystic Nephroma: A Case Report. 2008;52:91-93

Chen KTK. Fine Needle Aspiration Cytology of Intraosseous Ganglion: A Case Report. 2008;52:451-453

Chen L (see Weber et al). 2008;52:320-324

Choi Y-J (see Jung et al). 2008;52:313-319

Chon S-H (see Jang et al). 2008;52:394-396 (Letter)

Chung MJ (see Lee et al). 2008;52:665-670

Ciatto S (see Maccallini et al). 2008;52:568-574

Ciocca V (see Jacob-Ampuero et al). 2008;52:687-690

Clua A (see Saro et al). 2008;52:87-90

Collins BT, Phillips NJ, Hsueh EC. Fine Needle Aspiration Biopsy of Splenic Hamartoma with Bi-zarre Stromal Cells: A Case Report. 2008;52:347-350

Confortini M (see Maccallini et al). 2008;52:568-574

Conley RC (see Pacheco et al). 2008;52:575-578

Correia C (see Barroca et al). 2008;52:384-386 (Letter)

Cosar E (see Yantiss et al). 2008;52:133-138

Crapanzano JP (see Qiu et al). 2008;52:139-144

\section{D}

Dall' Agnol M (see Utagawa et al). 2008;52:439-444

Dalla Palma P (see Bonzanini et al). 2008;52:541-548

Daneshbod K (see Daneshbod et al). 2008;52:72-76

Daneshbod K (see Daneshbod et al). 2008;52:268-270 (Letter)

Daneshbod K (see Daneshbod et al). 2008;52:387-389 (Letter)

Daneshbod Y, Arabi MA, Ramzi M, Daneshbod K. Jaw Lesion as the First Presentation of Multiple Myeloma Diagnosed by Fine Needle Aspiration. 2008;52:268-270 (Letter)

Daneshbod Y, Daneshbod K, Negahban S, Aledavud A, Taghavi A, Saberi-Firuzi M, Fattahi MR, Salahi
H. Intraoperative Touch Imprint Diagnosis of Strongyloides stercoralis Infection Mimicking a Malignant Tumor. 2008;52:387-389 (Letter)

Daneshbod Y, Daneshbod K, Rasekhi A-R, Mosayebi Z, Negahban S, Hodjati S-R, Bedayat GR, GanjeiAzar P. Cytologic Differentiation of Struma Ovarii from Other Ovarian Neoplasms. 2008;52:72-76

Daneshbod Y, Khademi B. Chondromyxoid Fibroma of the Mandible: A Diagnostic Pitfall on Aspiration Cytology of Parotid. 2008;52:636-638 (Letter)

Daneshbod Y, Khademi B, Kadivar M, Ganjei-Azar P. Fine Needle Aspiration of Salivary Gland Lesions with Multinucleated Giant Cells. 2008;52: 671-680

Das DK, Rifaat AA, George SS, Grover VK, Mathew TC, Mirza K. Morphologic Changes in Fibroadenoma of Breast Due to Chickenpox: A Case Report with Suspicious Cytology in Fine Needle Aspiration Smears. 2008;52:337-343

Das K (see Fitzhugh et al). 2008;52:240-246

Dávila RM (see Zhai et al). 2008;52:196-200

Dawsey SM (see Pan et al). 2008;52:14-23

De Chiara A (see Fulciniti et al). 2008;52:612-618

de Llano P (see Santamaría and de Llano). 2008;52: 215-219

De Renne L (see Proca et al). 2008;52:83-86

de Wilde PC (see Grefte et al). 2008;52:35-44

Dejmek A. CK5/6 in Effusions: No Difference Between Mesothelioma and Pulmonary and Nonpulmonary Adenocarcinoma. 2008;52:579-583

Delatour NLDR (see Mokhtar et al). 2008;52:169-177

Demir N, Altay M, Özer E, Ünlü N, Duranay M, Üstün H, Duman S. Duration of Renal Failure as Risk Factor for Conjunctival Squamous Metaplasia. 2008;52:309-312

Demuru A (see Fulciniti et al). 2008;52:178-186

Derchain S (see Syrjänen et al). 2008;52:641-653

Desai M (see McGrath et al). 2008;52:351-353

Destouni CT (see Sioutopoulou et al). 2008;52:304-308

Dey P (see Bhatia et al). 2008;52:329-333

Dhimes P (see González et al). 2008;52:490-494

Dhurandhar B (see Steinman et al). 2008;52:279-285

Di Filippo L (see Steinman et al). 2008;52:279-285

Di Gabriele G (see Maccallini et al). 2008;52:568-574

di Loreto C (see Utagawa et al). 2008;52:439-444

Di Mattia D (see Fulciniti et al). 2008;52:612-618

Digumarti RR (see Prayaga et al). 2008;52:702-709

Duman S (see Demir et al). 2008;52:309-312

Duranay M (see Demir et al). 2008;52:309-312

\section{$\mathbf{E}$}

Eghbali S (see Mostaghni et al). 2008;52:597-601

Ehya H (see Young et al). 2008;52:277-278 
Ekici Ö (see Tokyol et al). 2008;52:235-239

Erhan Y (see Akbulut et al). 2008;52:65-71

Eržen J (see Terčelj et al). 2008;52:584-590

Esteva E (see Saro et al). 2008;52:87-90

Evans TN (see Carter et al). 2008;52:459-463

Eves M (see Carter et al). 2008;52:725-728

\section{$\mathbf{F}$}

Farag R, Redline R, Abdul-Karim FW. Value of Combining HPV-DNA Testing with Follow-up Papanicolaou Smear in Patients with Prior Atypical Squamous Cells of Undetermined Significance. 2008;52:294-296

Fattahi MR (see Daneshbod et al). 2008;52:387-389 (Letter)

Feng J (see Vella et al). 2008;52:377-378 (Letter)

Feng J, Al-Abbadi MA, Bandyopadhyay S, Salimnia H, Husain M. Significance of High-Risk Human Papillomavirus DNA-Positive Atypical Squamous Cells of Undetermined Significance Pap Smears in Perimenopausal and Postmenopausal Women. 2008; 52:434-438

Feoli F, Paesmans M, Van Eeckhout P. Fine Needle Aspiration Cytology of the Breast: Impact of Experience on Accuracy, Using Standardized Cytologic Criteria. 2008;52:145-151

Fischer AH (see Yantiss et al). 2008;52:133-138

Fitzhugh VA, Mirani N, Aisner S, Koneru B, Das K. Preoperative Fine Needle Aspiration Cytology Diagnosis of Microcystic Adenoma of the Pancreas: Fact or Fiction? A Report of 2 Cases. 2008;52:240246

Florez L (see Takei et al). 2008;52:445-450

Fokter SK (see Repše-Fokter and Fokter). 2008;52:8-13

Fortunato C (see Maccallini et al). 2008;52:568-574

Frangella C (see Fulciniti et al). 2008;52:178-186

Frías C (see Yuste et al). 2008;52:191-195

Frost F (see Wood et al). 2008;52:412-417

Fuchinoue F (see Komatsu et al). 2008;52:591-596

Fukudome N (see Kojima et al). 2008;52:467-470

Fulciniti F, Di Mattia D, Bove P, Mastro AA, De Chiara A, Botti G, Petrillo A, Apice G. Fine Needle Aspiration of Metastatic Epithelioid Angiosarcoma: A Report of 2 Cases. 2008;52:612-618

Fulciniti F, Frangella C, Staiano M, La Vecchia F, Botti G, Demuru A, Magliulo G, Boon ME. AirDried Smears for Optimal Diagnostic Immunocytochemistry. 2008;52:178-186

\section{G}

Gabhane SK, Gangane N, Anshu. Cytodiagnosis of
Eumycotic Mycetoma: A Case Report. 2008;52: 354-356

Gangane N (see Gabhane et al). 2008;52:354-356

Gangane N, Anshu, Singh R. Role of Modified Bleach Method in Staining of Acid-Fast Bacilli in Lymph Node Aspirates. 2008;52:325-328

Gangane N, Kamra H, Anshu, Joshi D, Gupta A. Cytodiagnosis of Granulocytic Sarcoma of Liver Arising in a Milieu of Myeloid Metaplasia: A Case Report. 2008;52:619-622

Ganjei-Azar P (see Daneshbod et al). 2008;52:72-76

Ganjei-Azar P (see Daneshbod et al). 2008;52:671680

Garbar C, Remmelink M, Mascaux C. Fine Needle Aspiration Cytology of Lymph Node: Experience of 2 University Hospitals with Conventional Smears and Liquid-Based Cytology. 2008;52:418-423

Garbyal RS, Arundhati, Bohra A, Bhujun KH. An Unusual Presentation of Filariasis: A Case Report. 2008;52:204-206

Garbyal RS, Gupta P, Bohra A, Gupta A, Kumar S. Tibial Giant Cell Tumor with Distant Subcutaneous Metastasis. 2008;52:270-273 (Letter)

García-Prats MD (see Vera-Alvarez et al). 2008;52: 264-266 (Letter)

García-Solano J (see Pérez-Guillermo et al). 2008;52: 259-260 (Letter)

Geisinger KR (see Patel et al). 2008;52:454-463

George SS (see Das et al). 2008;52:337-343

George SS (see Kapila et al). 2008;52:681-686

Geramizadeh B, Mirbahari Q, Taghavi A. Diagnosis of Giardiasis by Common Bile Duct Brushing Cytology. 2008;52:514-515 (Letter)

Gerasimidou D (see Sioutopoulou et al). 2008;52:304308

Ghosh A, Arundhati, Asthana AK. Pleomorphic Adenoma of the Parotid Gland Metastasizing to the Scapular Region: A Case Report. 2008;52:733-735

Giffen CA (see Pan et al). 2008;52:14-23

Gilliatt MA (see Mokhtar et al). 2008;52:169-177

Goel D (see Babu et al). 2008;52:464-466

Goel D, Babu S, Prayaga AK, Sundaram C. Pulmonary Metastases of Recurrent Intracranial Hemangiopericytoma Diagnosed on Fine Needle Aspiration Cytology: A Case Report. 2008;52:344-346

Goel D, Prayaga AK, Sundaram C, Raghunadharao D, Rajappa SJ, Rammurti S, Rao TM, Kumar RV. Utility of Fine Needle Aspiration Cytology in Mediastinal Lesions: A Clinicopathologic Study of 161 Cases from a Single Institution. 2008;52:404411

Goel K, Gupta S, Pai MR, Naik R. Comparison of Diagnostic Accuracy of Cytologic Sampling Techniques in Lung Cancer: A Study of 541 Cases from Coastal Karnataka. 2008;52:638-639 (Letter) 
Goel MM, Budhwar P. Fine Needle Aspiration Cytology and Immunocytochemistry in Tuberculous Thyroiditis: A Case Report. 2008;52:602-606

Goel MM, Budhwar P. Species-Specific Immunocytochemical Localization of Mycobacterium tuberculosis Complex in Fine Needle Aspirates of Tuberculous Lymphadenitis Using Antibody to $38 \mathrm{kDa}$ Immunodominant Protein Antigen. 2008;52:424433

Gokaslan T (see Patino et al). 2008;52:718-720

Goneppanavar M (see Siddaraju et al). 2008;52:495499

González ET, Argüelles M, Jiménez-Heffernan JA, Dhimes P, Vicandi B, Pinedo F. Cytologic Features of Hepatoid Carcinoma of the Ovary: A Case Report with Immunocytologic Evaluation of HepPar1. 2008; 52:490-494

González ET, Sánchez-Yuste R, Jiménez-Heffernan JA. Cytologic Features of Pulmonary Alveolar Adenoma. 2008;52:739-740 (Letter)

Gottimukkala SR (see Prayaga et al). 2008;52:702-709

Grafstein N (see Al-Agha et al). 2008;52:94-98

Grapsa D (see Athanassiadou and Grapsa). 2008;52: 24-34

Greening SE (see Young et al). 2008;52:277-278

Grefte JMM, de Wilde PC, Salet-van de Pol MRJ, Tomassen M, Raaymakers-van Geloof WL, Bulten J. Improved Identification of Malignant Cells in Serous Effusions Using a Small, Robust Panel of Antibodies on Paraffin-Embedded Cell Suspensions. 2008;52:35-44

Grieco V (see Zhu and Grieco). 2008;52:535-540

Griesshammer M (see Bangerter et al). 2008;52:52-55

Grover VK (see Das et al). 2008;52:337-343

Gu M (see Wardeh and Gu). 2008;52:481-484

Gulbahce HE (see Thrall et al). 2008;52:1-7

Guo H-Q (see Pan et al). 2008;52:14-23

Gupta A (see Gangane et al). 2008;52:619-622

Gupta A (see Garbyal et al). 2008;52:270-273 (Letter)

Gupta P (see Garbyal et al). 2008;52:270-273 (Letter)

Gupta P (see Young et al). 2008;52:277-278

Gupta R (see Mathur et al). 2008;52:740-743 (Letter)

Gupta R, Singh S. Cytologic Diagnosis of Fibrous Hamartoma of Infancy: A Case Report of a Rare Soft Tissue Lesion. 2008;52:201-203

Gupta RJ (see Kishore et al). 2008;52:710-712

Gupta S (see Goel et al). 2008;52:638-639 (Letter)

Gupta S (see Halder et al). 2008;52:286-293

\section{H}

Ha H-J (see Jin et al). 2008;52:357-360

Haas AR (see Jacob-Ampuero et al). 2008;52:687-690

Haba R (see Ohsaki et al). 2008;52:297-303
Haji BE (see Kapila et al). 2008;52:681-686

Halder K, Chachra KL, Sodhani P, Gupta S. Utility of Imprint Cytology for Early Presumptive Diagnosis in Clinically Suspicious Cervical Cancer. 2008;52:286-293

Hammes LS (see Syrjänen et al). 2008;52:641-653

Han L (see Vella et al). 2008;52:377-378 (Letter)

Han YM (see Lee et al). 2008;52:665-670

Handa U (see Batra et al). 2008;52:77-82

Hao C-Q (see Pan et al). 2008;52:14-23

Harbour JW (see Zhai et al). 2008;52:196-200

Hayati JN (see Jayaram et al). 2008;52:119-121 (Letter)

Heinrich B (see Bangerter et al). 2008;52:52-55

Henry M (see Pan et al). 2008;52:14-23

Hirakawa E (see Ohsaki et al). 2008;52:297-303

Hodjati S-R (see Daneshbod et al). 2008;52:72-76

Hsieh M-H, Lin M-C, Shun C-T, Chang T-C. Fine Needle Aspiration Cytology of Mixed MedullaryFollicular Thyroid Carcinoma: A Case Report. 2008; 52:361-365

Hsu C-Y (see Lai et al). 2008;52:563-567

Hsueh EC (see Collins et al). 2008;52:347-350

Humphrey SK (see Caudill et al). 2008;52:45-51

Husain M (see Feng et al). 2008;52:434-438

\section{I}

Iliyasu Y (see Malami and Iliyasu). 2008;52:400-403

Inayama Y (see Kanazawa et al). 2008;52:266-268 (Letter)

Iseki M (see Kojima et al). 2008;52:467-470

Ishii M (see Kanazawa et al). 2008;52:266-268 (Letter)

Islam S (see Mokhtar et al). 2008;52:169-177

Iyengar KR (see Basu et al). 2008;52:211-214

Iyengar KR (see Siddaraju et al). 2008;52:391-394 (Letter)

\section{J}

Jacob-Ampuero M-P, Haas AR, Ciocca V, Bibbo M. Cytologic Accuracy of Samples Obtained by Endobronchial Ultrasound-Guided Transbronchial Needle Aspiration at Thomas Jefferson University Hospital. 2008;52:687-690

Jagdish S (see Basu et al). 2008;52:211-214

Jain S (see Batra et al). 2008;52:152-158

Jain S (see Sachdev et al). 2008;52:511-512 (Letter)

Jain S (see Varma et al). 2008;52:549-556

Jang K-S (see Jang et al). 2008;52:394-396 (Letter)

Jang S-H, Jang K-S, Min K-W, Paik SS, Chon S-H.

Ruptured Thymic Teratoma Diagnosed by Pleural

Effusion Cytology. 2008;52:394-396 (Letter) 
Jashnani KD (see Kamat et al). 2008;52:517-519 (Letter) Jayaram G, Hayati JN, Yip CH, Ranganathan S, Taib NA. Cytologic, Histologic and Immunocytochemical Features in Fine Needle Aspiration of Paraganglioma-like Variant of Medullary Carcinoma. 2008;52:119-121 (Letter)

Jha RS (see Thapliyal et al). 2008;52:627-630

Jiménez-Heffernan JA (see González et al). 2008;52: 490-494

Jiménez-Heffernan JA (see González et al). 2008;52: 739-740 (Letter)

Jin GY (see Lee et al). 2008;52:665-670

Jin M-S, Ha H-J, Baek HJ, Lee JC, Koh JS. Adenomyomatous Hamartoma of Lung Mimicking Benign Mucinous Tumor in Fine Needle Aspiration Biopsy: A Case Report. 2008;52:357-360

Joshi D (see Gangane et al). 2008;52:619-622

Joshi U (see Thapliyal et al). 2008;52:627-630

Jung C-K, Lee A, Jung E-S, Choi Y-J, Jung S-L, Lee

K-Y. Split Sample Comparison of a Liquid-Based

Method and Conventional Smears in Thyroid Fine

Needle Aspiration. 2008;52:313-319

Jung E-S (see Jung et al). 2008;52:313-319

Jung S-L (see Jung et al). 2008;52:313-319

\section{K}

Kadivar M (see Daneshbod et al). 2008;52:671-680

Kamat RN, Jashnani KD, Rege JD. Cytologic Findings of an Uncommon Rosette-Forming Osteosarcoma. 2008;52:517-519 (Letter)

Kaminsky DB. Into Africa: Cytology for One World. 2008;52:399

Kampas LI (see Sioutopoulou et al). 2008;52:304-308

Kamra H (see Gangane et al). 2008;52:619-622

Kanazawa M, Ishii M, Sato Y, Kitamura K, Oshiro H, Inayama Y. Capsule-Deficient Meningeal Cryptococcosis. 2008;52:266-268 (Letter)

Kanjanavirojkul N, Kularbkaew C, Yutanawiboonchai W. Fine Needle Aspiration in a Malignant Lymphoepithelial Lesion: A Case Report. 2008;52:369372

Kapila K, Pathan SK, Al-Mosawy FA, George SS, Haji BE, Al-Ayadhy B. Fine Needle Aspiration Cytology of Breast Masses in Children and Adolescents: Experience with 1,404 Aspirates. 2008;52: 681-686

Kapkac M (see Akbulut et al). 2008;52:65-71

Kapkac M (see Akbulut et al). 2008;52:99-104

Kattoor J (see Ramadas et al). 2008;52:396-398 (Letter)

Kaur J (see Vankalakunti et al). 2008;52:729-732

Keith J, Ang L-C, Megyesi J. Cytodiagnosis of Supratentorial Hemangioblastoma. 2008;52:260-263 (Letter)
Kemp AMC, Bukvic M, Sturgis CD. Fine Needle Aspiration Diagnosis of Osteitis Fibrosa Cystica (Brown Tumor of Bone): A Case Report. 2008;52: 471-474

Keyhani-Rofagha S (see Proca et al). 2008;52:83-86

Khademi B (see Ashraf et al). 2008;52:745-747 (Letter)

Khademi B (see Daneshbod and Khademi). 2008;52: 636-638 (Letter)

Khademi B (see Daneshbod et al). 2008;52:671-680

Khader SN (see Al-Agha et al). 2008;52:94-98

Khandelwal N (see Bhatia et al). 2008;52:523-529

Khare P (see Kishore et al). 2008;52:710-712

Khunamornpong S (see Charoenkwan et al). 2008;52: 654-658

Kilpatrick SE (see Li et al). 2008;52:607-611

Kini S, Saraf CK, Naik LP, Shah VB, Puranik GV, Vartakvi PK. Occult Medullary Carcinoma of Thyroid with Lymph Node Metastases: A Case Report. 2008;52:105-108

Kishore B, Khare P, Gupta RJ, Bisht SP. Microfilaria of Wuchereria bancrofti in Cytologic Smears: A Report of 5 Cases with Unusual Presentations. 2008;52:710-712

Kitamura K (see Kanazawa et al). 2008;52:266-268 (Letter)

Kitsiou E (see Pappa et al). 2008;52:485-489

Kiyomoto H (see Ohsaki et al). 2008;52:297-303

Kjeldahl K (see Thrall et al). 2008;52:1-7

Kochman ML (see Pan et al). 2008;52:14-23

Koh JS (see Jin et al). 2008;52:357-360

Kojima H, Mori K, Fukudome N, Iseki M, Shimizu S. Cytologic Characteristics of Intracytoplasmic Refractile Eosinophilic Granular Bodies in Anaplastic Oligodendroglioma: A Case Report. 2008;52:467-470

Komatsu A (see Komatsu et al). 2008;52:591-596

Komatsu K, Nakanishi Y, Seki T, Yoshino A, Fuchinoue F, Amano S, Komatsu A, Sugitani M, Nemoto N. Application of Liquid-Based Preparation to Fine Needle Aspiration Cytology in Breast Cancer. 2008;52:591-596

Koneru B (see Fitzhugh et al). 2008;52:240-246

Krishnanand G (see Sharma and Krishnanand). 2008; 52:56-64

Kularbkaew C (see Kanjanavirojkul et al). 2008;52:369-372

Kumar A (see Babu et al). 2008;52:464-466

Kumar Behera S, Patro M, Mishra D, Bal A, Behera B, Sahoo S. Fine Needle Aspiration in Aspergilloma of Frontal Sinus: A Case Report. 2008;52:500-504

Kumar N (see Batra et al). 2008;52:152-158

Kumar P (see Mostaghni et al). 2008;52:597-601

Kumar PV (see Shamsi et al). 2008;52:187-190

Kumar R (see Anand et al). 2008;52:251-254

Kumar RV (see Goel et al). 2008;52:404-411

Kumar S (see Garbyal et al). 2008;52:270-273 (Letter)

Kumar S (see Vankalakunti et al). 2008;52:639-640 (Letter) 
$\mathbf{L}$

La Vecchia F (see Fulciniti et al). 2008;52:178-186

Lai C-R, Hsu C-Y, Tsay S-H, Li AF-Y. Clinical Significance of Atypical Glandular Cells by the 2001 Bethesda System in Cytohistologic Correlation. 2008;52:563-567

Lattanzi A (see Maccallini et al). 2008;52:568-574

Layfield LJ, Skripenova S, Bentz J, Smock C. Cytologic Fine Needle Aspiration Findings by Vegetant Intravascular Hemangioendothelioma. 2008;52: 273-275 (Letter)

Lee A (see Jung et al). 2008;52:313-319

Lee CY-K, Ng W-K. Follow-up Study of Atypical Glandular Cells in Gynecologic Cytology Using Conventional Pap Smears and Liquid-Based Preparations: Impact of the Bethesda System 2001. 2008;52:159-168

Lee JC (see Jin et al). 2008;52:357-360

Lee K-Y (see Jung et al). 2008;52:313-319

Lee YS, Jin GY, Han YM, Chung MJ, Park HS. Computed Tomography-Guided Transthoracic Needle Aspiration Biopsy of Intrapulmonary Lesions: Utility of a Liquid-Based Cytopreparatory Technique. 2008;52:665-670

Leiman G (see Sherman et al). 2008;52:659-664

Lerma E (see Saro et al). 2008;52:87-90

Li AF-Y (see Lai et al). 2008;52:563-567

Li HC, Roux JJ, Pranikoff T, Kilpatrick SE, Cappellari JO. Deep Juvenile Xanthogranuloma Presenting as a Chest Wall Mass: A Case Report. 2008;52: 607-611

Lichtenberger G (see Pogany et al). 2008;52:228-230

Lima CF (see Almeida et al). 2008;52:697-701

Lin M-C (see Hsieh et al). 2008;52:361-365

Longatto-Filho A (see Syrjänen et al). 2008;52:641653

Longatto-Filho A (see Utagawa et al). 2008;52:439_ 444

López A (see Yuste et al). 2008;52:191-195

López-López JI (see Vera-Alvarez et al). 2008;52:264266 (Letter)

López-Presa D (see Catalina-Fernández et al). 2008; 52:380-381 (Letter)

Loya A (see Prayaga et al). 2008;52:702-709

Lu N (see Pan et al). 2008;52:14-23

Lynch M (see McGrath et al). 2008;52:351-353

\section{M}

Maccallini V, Angeloni C, Caraceni D, Fortunato C, Venditti MA, Di Gabriele G, Antonelli C, Lattanzi A, Puliti D, Ciatto S, Confortini M, Sani C, Zappa M. Comparison of the Conventional Cervical
Smear and Liquid-Based Cytology: Results of a Controlled, Prospective Study in the Abruzzo Region of Italy. 2008;52:568-574

MacIntosh RF, Merrimen JL, Barnes PJ. Application of the Probabilistic Approach to Reporting Breast Fine Needle Aspiration in Males. 2008;52:530-534 Maddali LS (see Prayaga et al). 2008;52:702-709 Magliulo G (see Fulciniti et al). 2008;52:178-186

Malami SA, Iliyasu Y. Fine Needle Aspiration Cytology in Nigeria. 2008;52:400-403

Malamou-Mitsi V (see Pappa et al). 2008;52:485-489

Malhotra P (see Sachdeva et al). 2008;52:623-626

Mandal S (see Varma et al). 2008;52:549-556

Manucha V, Sun C-CJ. Cytologic Findings and Differential Diagnosis in Hepatic Epithelioid Hemangioendothelioma: A Case Report. 2008;52:713-717

Manucha V, Zhao F, Rodgers W. Atypical Lymphoid Cells in Cerebrospinal Fluid in Acute Epstein Barr Virus Infection: A Case Report Demonstrating a Pitfall in Cerebrospinal Fluid Cytology. 2008;52: 334-336

Marigil-Gómez M (see Vera-Alvarez et al). 2008;52: 264-266 (Letter)

Marques C (see Barroca et al). 2008;52:124-132

Marsh WL Jr (see Proca et al). 2008;52:83-86

Mathur SR, Aron M, Gupta R, Sharma MC, Arora VK. Malignant Mesothelioma of Tunica Vaginalis: A Report of 2 Cases with Preoperative Cytologic Diagnosis. 2008;52:740-743 (Letter)

Martín P (see Yuste et al). 2008;52:191-195

Mascaux C (see Garbar et al). 2008;52:418-423

Mastro AA (see Fulciniti et al). 2008;52:612-618

Mathew TC (see Das et al). 2008;52:337-343

Mathews A (see Ramadas et al). 2008;52:396-398 (Letter)

Matsunaga T (see Ohsaki et al). 2008;52:297-303

Mattosinho de Castro Ferraz MdG (see Utagawa et al). 2008;52:439-444

McGrath SM, Rana DN, Lynch M, Desai M. Metastatic Transitional Cell Carcinoma Causing a Unilateral Pleural Effusion: A Case Report. 2008;52: 351-353

Megyesi J (see Keith et al). 2008;52:260-263 (Letter)

Merrimen JL (see MacIntosh et al). 2008;52:530-534

Miller MJ, Cazzaniga M, Casadio C. Cytologic Findings of Breast Ductal Lavage and Concurrent Fine Needle Aspiration in Pleomorphic Lobular Carcinoma in Situ: A Case Report. 2008;52:207-210

Min K-W (see Jang et al). 2008;52:394-396 (Letter)

Mirani N (see Fitzhugh et al). 2008;52:240-246

Mirbahari Q (see Geramizadeh et al). 2008;52:514515 (Letter)

Mirza K (see Das et al). 2008;52:337-343

Mishra D (see Behera et al). 2008;52:635-636 (Letter)

Mishra D (see Kumar Behera et al). 2008;52:500-504

Mishra MM (see Siddaraju et al). 2008;52:495-499 
Mishra MN (see Tewari et al). 2008;52:743-745 (Letter) Misra V, Singh PA. Cytodiagnosis of Extraosseous Mesenchymal Chondrosarcoma of Meninges: A Case Report. 2008;52:366-368

Mody DR (see Steinman et al). 2008;52:279-285

Mohan H (see Batra et al). 2008;52:77-82

Mokhtar GA, Delatour NLDR, Assiri AH, Gilliatt MA, Senterman M, Islam S. Atypical Squamous Cells, Cannot Exclude High-Grade Squamous Intraepithelial Lesion: Cytohistologic Correlation Study with Diagnostic Pitfalls. 2008;52:169-177

Montazer NR (see Shamsi et al). 2008;52:187-190

Mori K (see Kojima et al). 2008;52:467-470

Mosayebi Z (see Daneshbod et al). 2008;52:72-76

Mostaghni AA, Afarid M, Eghbali S, Kumar P. Evaluation of Brushing Cytology in the Diagnosis of Helicobacter pylori Gastritis. 2008;52:597-601

\section{$\mathbf{N}$}

Nagy GK, Newton LE. Comments on "Scientific Issues Related to the Cytology Proficiency Testing Regulations." 2008;52:117-118 (Letter)

Nahal A (see Brimo and Nahal). 2008;52:721-724

Naik LP (see Kini et al). 2008;52:105-108

Naik R (see Goel et al). 2008;52:638-639 (Letter)

Nakamura M (see Ohsaki et al). 2008;52:297-303

Nakanishi Y (see Komatsu et al). 2008;52:591-596

Nathan MH (see Sherman et al). 2008;52:659-664

Naud S (see Sherman et al). 2008;52:659-664

Nayak S (see Behera et al). 2008;52:635-636 (Letter)

Naylor R (see Rust et al). 2008;52:475-480

Negahban S (see Daneshbod et al). 2008;52:72-76

Negahban S (see Daneshbod et al). 2008;52:387-389 (Letter)

Nelson JJ (see Carter et al). 2008;52:725-728

Nemoto N (see Komatsu et al). 2008;52:591-596

Newton LE (see Nagy and Newton). 2008;52:117118 (Letter)

$\mathrm{Ng} \mathrm{W-K} \mathrm{(see} \mathrm{Lee} \mathrm{and} \mathrm{Ng).} \mathrm{2008;52:159-168}$

Nijhawan R (see Bhatia et al). 2008;52:329-333

Nijhawan R (see Bhatia et al). 2008;52:523-529

Nijhawan R (see Vankalakunti et al). 2008;52:639-640 (Letter)

Nijhawan VS (see Tewari et al). 2008;52:743-745 (Letter)

Nimmanahaeminda K (see Charoenkwan et al). 2008; $52: 654-658$

Ohsaki H, Haba R, Matsunaga T, Nakamura M, Kiyomoto H, Hirakawa E. Cytomorphologic and Im- munocytochemical Characteristics of Reactive Renal Tubular Cells in Renal Glomerular Disease. 2008;52:297-303

Ortega JA (see Pérez-Guillermo et al). 2008;52:259_ 260 (Letter)

Oshiro H (see Kanazawa et al). 2008;52:266-268 (Letter)

Ozdemir N (see Akbulut et al). 2008;52:65-71

Ozdemir N (see Akbulut et al). 2008;52:99-104

Özer E (see Demir et al). 2008;52:309-312

Özkara SK, Turan G. Cystic Fluid and Fine Needle Aspiration Cytopathology of Cystic Adult Granulosa Cell Tumor of the Ovary: A Case Report. 2008; 52:247-250

Pacheco MC, Conley RC, Pennington DW, Bishop JW. Concordance Between Original Screening and Final Diagnosis Using Imager vs. Manual Screen of Cervical Liquid-Based Cytology Slides. 2008;52: 575-578

Pacini P (see Palleschi et al). 2008;52:389-391 (Letter)

Paesmans M (see Feoli et al). 2008;52:145-151

Pai MR (see Goel et al). 2008;52:638-639 (Letter)

Paik SS (see Jang et al). 2008;52:394-396 (Letter)

Paioli D (see Trisolini et al). 2008;52:263-264 (Letter)

Palleschi GM, Torchia D, Pacini P. Ultrastructural Alterations of Superficial Keratinocytes in Bullous Pemphigoid as Detected by Scanning Electron Microscopy. 2008;52:389-391 (Letter)

Palmieri B, Sblendorio V, Saleh F, Al-Sebeih K. Securebox: A Multibiopsy Sample Container for Specimen Identification and Transport. 2008;52: 691-696

Pambuccian SE (see Thrall et al). 2008;52:1-7

Pan Q-J, Roth MJ, Guo H-Q, Kochman ML, Wang G-Q, Henry M, Wei W-Q, Giffen CA, Lu N, Abnet CC, Hao C-Q, Taylor PR, Qiao Y-L, Dawsey SM. Cytologic Detection of Esophageal Squamous Cell Carcinoma and Its Precursor Lesions Using Balloon Samplers and Liquid-Based Cytology in Asymptomatic Adults in Linxian, China. 2008;52:14-23

Pangarkar MA (see Wahane et al). 2008;52:381-384 (Letter)

Pappa L, Zagorianakou N, Kitsiou E, Sintou-Mantela E, Bafa M, Malamou-Mitsi V. Breast Metastasis from Uterine Leiomyosarcoma Diagnosed by Fine Needle Aspiration: A Case Report. 2008;52:485489

Park HS (see Lee et al). 2008;52:665-670

Patel NP, Bergman S, Geisinger KR. Fine-Needle Aspiration Biopsy Cytology of Cutaneous Calci- 
nosis in a 74-Year-Old Woman with Dermatomyositis on Methotrexate Therapy: A Case Report. 2008; 52:454-463

Patelli M (see Trisolini et al). 2008;52:263-264 (Letter)

Pathan SK (see Kapila et al). 2008;52:681-686

Patino WD, Cavuoti D, Banerjee SK, Swartz K, Ashfaq R, Gokaslan T. Cytologic Diagnosis of Blastocystis hominis in Peritoneal Fluid: A Case Report. 2008;52:718-720

Patro M (see Kumar Behera et al). 2008;52:500-504

Pennington DW (see Pacheco et al). 2008;52:575-578

Pérez-Guillermo M, García-Solano J, Ortega JA. Fine Needle Aspiration Cytology of Palpable Lesions of the Scrotal Content. 2008;52:259-260 (Letter)

Petrillo A (see Fulciniti et al). 2008;52:612-618

Phillips NJ (see Collins et al). 2008;52:347-350

Pinedo F (see González et al). 2008;52:490-494

Pogany P, Szucs E, Lichtenberger G, Vass L. Diagnosis of Myiasis by Fine Needle Aspiration Cytology: A Case Report. 2008;52:228-230

Pranikoff T (see Li et al). 2008;52:607-611

Prayaga A (see Babu et al). 2008;52:464-466

Prayaga AK (see Goel et al). 2008;52:344-346

Prayaga AK (see Goel et al). 2008;52:404-411

Prayaga AK, Loya A, Gottimukkala SR, Digumarti RR, Maddali LS, Challa S. Cytologic Features of Primary Malignant Tumors of Skin and Adnexae. 2008;52:702-709

Primic-Zakelj M (see Terčelj et al). 2008;52:584-590

Proca DM, De Renne L, Marsh WL Jr, KeyhaniRofagha S. Anaplastic Large Cell Lymphoma in a Human Immunodeficiency Virus-Positive Patient with Cytologic Findings in Bladder Wash: A Case Report. 2008;52:83-86

Prodnik L (see Terčelj et al). 2008;52:584-590

Puliti D (see Maccallini et al). 2008;52:568-574

Puranik GV (see Kini et al). 2008;52:105-108

\section{Q}

Qiao Y-L (see Pan et al). 2008;52:14-23

Qiu L, Crapanzano JP, Saqi A, Vidhun R, Vazquez MF. Cell Block Alone as an Ideal Preparatory Method for Hemorrhagic Thyroid Nodule Aspirates Procured Without Onsite Cytologists. 2008; $52: 139-144$

\section{$\mathbf{R}$}

Raaymakers-van Geloof WL (see Grefte et al). 2008; $52: 35-44$
Raghunadharao D (see Goel et al). 2008;52:404-411

Raina V (see Anand et al). 2008;52:251-254

Rajappa SJ (see Goel et al). 2008;52:404-411

Rajwanshi A (see Bhatia et al). 2008;52:523-529

Ramadas PT, Kattoor J, Mathews A, Abraham EK. Fine Needle Aspiration Cytology of Langerhans Cell Thyroid Histiocytosis and Its Draining Lymph Nodes. 2008;52:396-398 (Letter)

Rammurti S (see Goel et al). 2008;52:404-411

Ramzi M (see Daneshbod et al). 2008;52:268-270 (Letter)

Rana DN (see McGrath et al). 2008;52:351-353

Ranganathan S (see Jayaram et al). 2008;52:119-121 (Letter)

Rao IS (see Babu et al). 2008;52:464-466

Rao TM (see Goel et al). 2008;52:404-411

Rasekhi A-R (see Daneshbod et al). 2008;52:72-76

Redline R (see Farag et al). 2008;52:294-296

Rege JD (see Kamat et al). 2008;52:517-519 (Letter)

Remmelink M (see Garbar et al). 2008;52:418-423

Repše-Fokter A, Fokter SK. Osteopenia and Osteoporosis Can Be Predicted from Pap Test. 2008;52: 8-13

Rifaat AA (see Das et al). 2008;52:337-343

Rodgers W (see Manucha et al). 2008;52:334-336

Roteli-Martins C (see Syrjänen et al). 2008;52:641653

Roth MJ (see Pan et al). 2008;52:14-23

Rott T (see Terčelj et al). 2008;52:584-590

Roux JJ (see Li et al). 2008;52:607-611

Roy S (see Sachdev et al). 2008;52:511-512 (Letter)

Roy SK (see Siddaraju et al). 2008;52:109-113

Rust MM, Susa J, Naylor R, Cavuoti D. Clear Cell Carcinoma in a Background of Endometriosis: Case Report of a Finding in a Midline Abdominal Scar 5 Years After a Total Abdominal Hysterectomy. 2008;52:475-480

Rylander R (see Terčelj et al). 2008;52:584-590

\section{S}

Saberi-Firuzi M (see Daneshbod et al). 2008;52:387389 (Letter)

Sachdev A (see Batra et al). 2008;52:77-82

Sachdev R, Roy S, Jain S. Tubercular Epididymoorchitis Masquerading as Testicular Malignancy: An Interesting Case. 2008;52:511-512 (Letter)

Sachdeva MUS, Suri V, Malhotra P, Srinivasan R. Cerebrospinal Fluid Infiltration in Hodgkin Lymphoma: A Case Report. 2008;52:623-626

Sáenz-Santamaria J (see Catalina-Fernández et al). 2008; 52:380-381 (Letter)

Sagramoso C (see Bonzanini et al). 2008;52:541-548

Sahoo S (see Kumar Behera et al). 2008;52:500-504 
Salahi H (see Daneshbod et al). 2008;52:387-389 (Letter)

Saleh F (see Palmieri et al). 2008;52:691-696

Salet-van de Pol MRJ (see Grefte et al). 2008;52:35-44

Salimnia H (see Feng et al). 2008;52:434-438

Salomão DR (see Caudill et al). 2008;52:45-51

Sánchez-Yuste R (see González et al). 2008;52:739_ 740 (Letter)

Sani C (see Maccallini et al). 2008;52:568-574

Santamaría M, de Llano P. Adenoid Cystic Carcinoma Metastatic to the Kidney: A Case Report. 2008; $52: 215-219$

Saqi A (see Qiu et al). 2008;52:139-144

Saraf CK (see Kini et al). 2008;52:105-108

Sarian L (see Syrjänen et al). 2008;52:641-653

Saro F, Clua A, Esteva E, Carreras A, Adán A, Lerma E. Cytologic Diagnosis of Ocular Melanocytoma: A Case Report. 2008;52:87-90

Sato Y (see Kanazawa et al). 2008;52:266-268 (Letter)

Satyanarayana S (see Tewari et al). 2008;52:743-745 (Letter)

Savik K (see Thrall et al). 2008;52:1-7

Sayana A (see Thapliyal et al). 2008;52:627-630

Sblendorio V (see Palmieri et al). 2008;52:691-696

Scheiber-Pact M (see Steinman et al). 2008;52:279_ 285

Schrembs I (see Bangerter et al). 2008;52:52-55

Seki T (see Komatsu et al). 2008;52:591-596

Senterman M (see Mokhtar et al). 2008;52:169-177

Setinek U (see Armbruster et al). 2008;52:223-227

Sever N (see Terčelj et al). 2008;52:584-590

Seymour AW (see Al-Agha et al). 2008;52:94-98

Sforza-Huffman C. Cytology of Fibrous Hamartoma of Infancy: A Helpful Approach for an Uncommon Soft Tissue Lesion. 2008;52:123

Shah VB (see Kini et al). 2008;52:105-108

Shamsi M, Abdali K, Montazer NR, Kumar PV, Tabatabaee HR. Comparison of Carnoy's Solution and 96\% Ethyl Alcohol Fixation in Bloody Pap Smears. 2008; 52:187-190

Sharma C, Krishnanand G. Histologic Analysis and Comparison of Techniques in Fine Needle Aspiration-Induced Alterations in Thyroid. 2008; 52:56-64

Sharma MC (see Mathur et al). 2008;52:740-743 (Letter)

Sharma S (see Anand et al). 2008;52:251-254

Sherman JF, Leiman G, Naud S, Nathan MH, Ambaye AB. Follicular and Hürthle Cell Lesions of the Thyroid: Can Inconclusive Results Be Minimized? 2008;52:659-664

Shifa R (see Bhatia et al). 2008;52:329-333

Shimizu S (see Kojima et al). 2008;52:467-470

Shirata NK (see Utagawa et al). 2008;52:439-444
Shishegar M (see Azarpira et al). 2008;52:220-222

Shun C-T (see Hsieh et al). 2008;52:361-365

Siddaraju N (see Basu et al). 2008;52:211-214

Siddaraju N, Badhe BA, Goneppanavar M, Mishra MM. Preoperative Fine Needle Aspiration Cytologic Diagnosis of Spindle Cell Myoepithelioma of the Parotid Gland: A Case Report. 2008;52:495499

Siddaraju N, Solo S, Badhe BA, Srinivasan R. Benign Skin Adenexal Tumor vs. Hemangioma: Diagnostic Pitfall Due to Cytologic Sampling Error. 2008;52:378-380 (Letter)

Siddaraju N, Solo S, Soundararaghavan J, Srinivasan R. Fine Needle Aspiration Biopsy in Pigmented Basal Cell Carcinoma. 2008;52:509-511 (Letter)

Siddaraju N, Solo S, Soundararaghavan J, Srinivasan R. Fine Needle Aspiration Biopsy of Pleomorphic Adenoma and Adenoid Cystic Carcinoma of the Lacrimal Gland. 2008;52:515-517 (Letter)

Siddaraju N, Soundararaghavan J, Bundele MM, Roy SK. Fine Needle Aspiration Cytology of Epithelioid Angiosarcoma: A Case Report. 2008;52:109113

Siddaraju N, Soundararaghavan J, Iyengar KR. Cytology of Pseudomyxoma Peritonei Associated with Well-Differentiated Appendiceal Adenocarcinoma. 2008;52:391-394 (Letter)

Singh DK (see Batra et al). 2008;52:152-158

Singh PA (see Misra and Singh). 2008;52:366-368

Singh R (see Gangane et al). 2008;52:325-328

Singh S (see Gupta and Singh). 2008;52:201-203

Sintou-Mantela E (see Pappa et al). 2008;52:485-489

Sioutopoulou DO, Kampas LI, Gerasimidou D, Valeri RM, Boukovinas I, Tsavdaridis D, Destouni CT. Diagnosis of Metastatic Tumors in Cerebrospinal Fluid Samples Using Thin-Layer Cytology. 2008;52:304-308

Sistla SC (see Basu et al). 2008;52:211-214

Skripenova S (see Layfield et al). 2008;52:273-275 (Letter)

Smith D (see Steinman et al). 2008;52:279-285

Smith ME (see Zhai et al). 2008;52:196-200

Smock C (see Layfield et al). 2008;52:273-275 (Letter)

Sodhani P (see Halder et al). 2008;52:286-293

Solo S (see Siddaraju et al). 2008;52:378-380 (Letter)

Solo S (see Siddaraju et al). 2008;52:509-511 (Letter)

Solo S (see Siddaraju et al). 2008;52:515-517 (Letter)

Soundararaghavan J (see Siddaraju et al). 2008;52: 109-113

Soundararaghavan J (see Siddaraju et al). 2008;52:391394 (Letter)

Soundararaghavan J (see Siddaraju et al). 2008;52: 509-511 (Letter)

Soundararaghavan J (see Siddaraju et al). 2008;52: 515-517 (Letter)

Squintani L (see Trisolini et al). 2008;52:263-264 (Letter) 
Srinivasan R (see Bhatia et al). 2008;52:329-333

Srinivasan R (see Bhatia et al). 2008;52:523-529

Srinivasan R (see Sachdeva et al). 2008;52:623-626

Srinivasan R (see Siddaraju et al). 2008;52:378-380 (Letter)

Srinivasan R (see Siddaraju et al). 2008;52:509-511 (Letter)

Srinivasan R (see Siddaraju et al). 2008;52:515-517

(Letter)

Srinivasan R (see Vankalakunti et al). 2008;52:729_ 732

Srinivasan S (see Tewari et al). 2008;52:743-745 (Letter)

Srisomboon J (see Charoenkwan et al). 2008;52:654658

Srivastava AK (see Thapliyal et al). 2008;52:627-630

Staiano M (see Fulciniti et al). 2008;52:178-186

Steinman S, Smith D, Chandler N, Dhurandhar B, Di Filippo L, Scheiber-Pact M, Mody DR. Morphologic, Patient and Interpreter Profiles of HighRisk Human Papillomavirus-Positive vs. -Negative Cases of Atypical Squamous Cells of Undetermined Significance. 2008;52:279-285

Sturgis CD (see Kemp et al). 2008;52:471-474

Sugitani M (see Komatsu et al). 2008;52:591-596

Sun C-CJ (see Manucha and Sun). 2008;52:713-717

Sun C-F (see Chang and Sun). 2008;52:231-234

Sundaram C (see Goel et al). 2008;52:344-346

Sundaram C (see Goel et al). 2008;52:404-411

Suri V (see Sachdeva et al). 2008;52:623-626

Susa J (see Rust et al). 2008;52:475-480

Swarbrick N (see Wood et al). 2008;52:412-417

Swartz K (see Patino et al). 2008;52:718-720

Syrjänen K, Derchain S, Roteli-Martins C, LongattoFilho A, Hammes LS, Sarian L, the Members of the Latin American Screening Study Group. Value of Conventional Pap Smear, Liquid-Based Cytology, Visual Inspection and Human Papillomavirus Testing as Optional Screening Tools Among Latin American Women $<35$ and $\geq 35$ Years of Age: Experience from the Latin American Screening Study. 2008;52:641-653

Szucs E (see Pogany et al). 2008;52:228-230

\section{$\mathbf{T}$}

Tabatabaee HR (see Shamsi et al). 2008;52:187-190

Taghavi A (see Daneshbod et al). 2008;52:387-389 (Letter)

Taghavi A (see Geramizadeh et al). 2008;52:514-515 (Letter)

Taib NA (see Jayaram et al). 2008;52:119-121 (Letter)

Takei H, Florez L, Bhattacharjee MB. Cytologic Features of Subependymal Giant Cell Astrocytoma: A Review of 7 Cases. 2008;52:445-450

Tavakol MH (see Ashraf et al). 2008;52:745-747 (Letter)
Taylor PR (see Pan et al). 2008;52:14-23

Terčelj M, Ales A, Rott T, Sever N, Prodnik L, Eržen J, Primic-Žakelj M, Rylander R. DNA-Based Sputum Cell Image Analysis for Lung Cancer in a Clinical Setting. 2008;52:584-590

Tewari R, Nijhawan VS, Mishra MN, Srinivasan S, Chattwal PK, Satyanarayana S. An Unusual Presentation of Type 2 Lepra Reaction: Role of Fine Needle Aspiration Cytology Foliaceous. 2008;52: 743-745 (Letter)

Thapliyal N, Joshi U, Vaibhav G, Sayana A, Srivastava AK, Jha RS. Pilomatricoma Mimicking Small Round Cell Tumor on Fine Needle Aspiration Cytology: A Case Report. 2008;52:627-630

Thorner PS (see Charoenkwan et al). 2008;52:654658

Thrall M, Kjeldahl K, Savik K, Gulbahce HE, Pambuccian SE. Rate of Endometrial Adenocarcinoma in Women Screened Before and After Implementation of the Bethesda 2001 Reporting System. 2008; $52: 1-7$

Tokyol Ç, Yılmaz MD, Ekici Ö, Aktepe F. Follicular Dendritic Cell Sarcoma: A Case Report. 2008;52: 235-239

Tomassen M (see Grefte et al). 2008;52:35-44

Torchia D (see Palleschi et al). 2008;52:389-391 (Letter)

Trisolini R, Paioli D, Patelli M, Cancellieri A, Squintani L. Bronchoalveolar Lavage: Intact Granulomas in Mycobacterium avium Pulmonary Infection. 2008; 52:263-264 (Letter)

Tsavdaridis D (see Sioutopoulou et al). 2008;52:304308

Tsay S-H (see Lai et al). 2008;52:563-567

Tucker JA (see Carter et al). 2008;52:459-463

Turan G (see Özkara and Turan). 2008;52:247-250

\section{U}

Ünlü N (see Demir et al). 2008;52:309-312

Uppal R (see Bhatia et al). 2008;52:329-333

Üstün H (see Demir et al). 2008;52:309-312

Utagawa ML, Shirata NK, Mattosinho de Castro Ferraz MdG, di Loreto C, Dall' Agnol M, LongattoFilho A. Performance of 3 Methods for Quality Control for Gynecologic Cytology Diagnoses. 2008; 52:439-444

\section{V}

Vaibhav G (see Thapliyal et al). 2008;52:627-630

Valeri RM (see Sioutopoulou et al). 2008;52:304308 
Vallejo C (see Yuste et al). 2008;52:191-195

Van Eeckhout P (see Feoli et al). 2008;52:145-151

Vankalakunti M, Kaur J, Srinivasan R. Cytology of Sarcoma Metastasizing to the Thyroid: A Case Report. 2008;52:729-732

Vankalakunti M, Kumar S, Nijhawan R. Microfilariae in Urine. 2008;52:639-640 (Letter)

Varma K, Jain S, Mandal S. Cytomorphologic Spectrum in Paraganglioma. 2008;52:549-556

Vartakvi PK (see Kini et al). 2008;52:105-108

Vasei M (see Ashraf et al). 2008;52:745-747 (Letter)

Vasishtha RK (see Bhatia et al). 2008;52:523-529

Vass L (see Pogany et al). 2008;52:228-230

Vazquez MF (see Qiu et al). 2008;52:139-144

Vella S, Al-Abbadi M, Han L, Feng J. Fine Needle Aspiration of Metastatic Undifferentiated Malignant Sex Cord Stromal Tumor. 2008;52:377-378 (Letter)

Venditti MA (see Maccallini et al). 2008;52:568-574

Venkataraman G (see Bargaje et al). 2008;52:512-514 (Letter)

Vera-Alvarez J, García-Prats MD, Marigil-Gómez M, Abascal-Agorreta M, López-López JI. Elastofibroma Dorsi Diagnosed by Fine Needle Aspiration Cytology. 2008;52:264-266 (Letter)

Verma SK (see Basu et al). 2008;52:211-214

Vicandi B (see González et al). 2008;52:490-494

Vidhun R (see Qiu et al). 2008;52:139-144

\section{w}

Wahane RN, Pangarkar MA, Bobhate SK. Fine Needle Aspiration Cytology of a Hydatid Cyst of the Pelvis and Hip Joint. 2008;52:381-384 (Letter)

Wang G-Q (see Pan et al). 2008;52:14-23

Wardeh R, Gu M. Cytologic Diagnosis of Hemophagocytic Lymphohistiocytosis in Ascitic Fluid: A Case Report. 2008;52:481-484

Weber D, Brainard J, Chen L. Atypical Epithelial Cells, Cannot Exclude Papillary Carcinoma, in Fine Needle Aspiration of the Thyroid. 2008;52: 320-324

Wei W-Q (see Pan et al). 2008;52:14-23

Wig J (see Bhatia et al). 2008;52:523-529

Wilbur DC (see Yuan and Wilbur). 2008;52:557562

Wojcik EM (see Bargaje et al). 2008;52:512-514 (Letter)

Wood B, Swarbrick N, Frost F. Diagnosis of Pulmonary Hamartoma by Fine Needle Biopsy. 2008; $52: 412-417$

\section{$\mathbf{X}$}

Xiao G-Q, Burstein DE. Cytologic Findings of Rhabdoid Meningioma in Cerebrospinal Fluid. 2008;52: 118-119 (Letter)

\section{$\mathbf{Y}$}

Yang GCH. Ultrasound-Guided Fine Needle Aspiration of the Pancreas: Endoscopic vs. Percutaneous Approach. 2008;52:521-522

Yantiss RK, Cosar E, Fischer AH. Use of IMP3 in Identification of Carcinoma in Fine Needle Aspiration Biopsies of Pancreas. 2008;52:133-138

Yilmaz MD (see Tokyol et al). 2008;52:235-239

Yip CH (see Jayaram et al). 2008;52:119-121 (Letter)

Yoshino A (see Komatsu et al). 2008;52:591-596

Young NA, Greening SE, Gupta P, Bibbo M, Ehya H. The Declining Pap Test: An Omen of Extinction or an Opportunity for Reform? 2008;52:277278

Yuan Q, Wilbur DC. Original Cervical Cytology and Follow-up Biopsy Results in Positive High Risk Human Papillomavirus DNA Tests with HighLevel Results. 2008;52:557-562

Yuste RS, Frías C, López A, Vallejo C, Martín P, Bellas C. Diagnostic Value of JC/BK Virus Antibody Immunohistochemistry Staining in Urine Samples from Posttransplant Immunosuppressed Patients in Relation to Polyomavirus Reactivation. 2008;52: 191-195

Yutanawiboonchai W (see Kanjanavirojkul et al). 2008; 52:369-372

$\mathbf{Z}$

Zagorianakou N (see Pappa et al). 2008;52:485-489

Zappa M (see Maccallini et al). 2008;52:568-574

Zekioglu O (see Akbulut et al). 2008;52:65-71

Zekioglu O (see Akbulut et al). 2008;52:99-104

Zhai J, Harbour JW, Smith ME, Dávila RM. Correlation Study of Benign Cytomorphology and Final Clinical Diagnosis. 2008;52:196-200

Zhao F (see Manucha et al). 2008;52:334-336

Zhu L-C, Grieco V. Diagnostic Value of Unusual Gross Appearance of Aspirated Material from Endoscopic Ultrasound-Guided Fine Needle Aspiration of Pancreatic and Peripancreatic Cystic Lesions. 2008;52:535-540 